\title{
Cosmological singularities in conformal Weyl gravity
}

\author{
Yaghoub Heydarzade ${ }^{\mathrm{a}}(\mathbb{D}$ \\ Department of Mathematics, Faculty of Sciences, Bilkent University, 06800 Ankara, Turkey
}

Received: 14 September 2019 / Accepted: 30 October 2019 / Published online: 14 November 2019

(C) The Author(s) 2019

\begin{abstract}
In this work, we study the issue of the past and future cosmological singularities in the context of the fourthorder conformal Weyl gravity. In particular, we investigate the emergent universe scenario proposed by Ellis et al., and find the stability conditions of the corresponding Einstein static state using the fixed point approach. We show that depending on the values of the parameters of the conformal Weyl gravity theory, there are possibilities for having initially stable emergent states for an FRW universe with both the positive and negative spatial curvatures. This represents that the conformal Weyl gravity can be free of the initial singularity problem. Then, following Barrow et al., we address the possible types of the finite-time future cosmological singularities. We discuss how these singularities also can be avoided in the context of this theory.
\end{abstract}

\section{Introduction}

The standard model of cosmology based on the Einstein general theory of relativity (GR) has a good agreement with the recent high resolution observations and provides an accurate overview of the cosmic history of our universe. However, it suffers from some fundamental problems such the initial big bang singularity problem, the flatness problem, the horizon problem, the magnetic monopoles and etc. Although the inflation scenario provides solutions for some of these problems, the initial big bang singularity problem still remains unsolved. Indeed, if the inflation scenario is realized by the dynamics of scalar fields coupled to Einstein gravity, then the Hawking-Penrose singularity theorem $[1,2]$ proves that an inflationary universe is geodesically incomplete in its past. Therefore, there exists a singularity before the onset of inflation and consequently the inflationary scenario is not capable to yield the complete history of the very early universe. To deal with this shortcoming, a number of attempts have been

a e-mail: yheydarzade@bilkent.edu.tr made in construction of cosmological models which are initially non-singular. There are two main proposals for this aim named as the "bouncing universe" and "emergent universe" scenarios. The bouncing universe scenario is based on a smooth transition of the universe at a finite radius from a contracting phase to an expanding phase. Then, by going backward in time, the universe collapses up to a finite value for the scale factor, before which it starts to expand again [3]. Although the bouncing scenario avoids the big bang singularity, the existence of the non-singular bounce requires non-standard matter fields violating energy conditions $[4,5]$. In the emergent universe scenario, the universe emerges from a static state, namely as the "Einstein static universe (ESU)", characterized by a non-zero spatial volume with a positive curvature [6,7]. This scenario avoids the initial big bang singularity while preserving the standard energy conditions. However, due to the existence of varieties of perturbations, such as the quantum fluctuations and the variations of the average energy density of the system [8], the emergent universe model is unstable and suffers from a fine-tuning problem [9]. This unstability problem can be amended by the modifications of the cosmological field equations of Einstein's GR. Indeed, there are two conditions which any gravitational theory should satisfy for admitting an emergent universe paradigm: (1) the existence and stability of the ESU, and (2) the possibility of joining the standard cosmological history by a graceful exit mechanism from the ESU. By this motivation, the emergent universe scenario and the stability issue of its Einstein static state has been explored in the context of various modified theories of gravity such as the loop quantum gravity [10,11], $f(R)$ gravity [12-14], $f(T)$ gravity [15-17], Einstein-Cartan theory $[18,19]$, Braneworlds [20-22] and Massive gravity [23,24] as well as in the presence of vacuum energy corresponding to conformally invariant fields [25] among the others. Most of the mentioned references just focused on the first condition without addressing the later one. 
On the other hand, after the discovery of the accelerating expansion of the universe, deeper studies of the features of the cosmic fluid responsible for this accelerating expansion, the so called "dark energy", showed the plethora of new types of singularities different than the initial big bang singularity. These new types of singularities are future singularities for a universe and they are characterized by the violation of some of the energy conditions. This violation in turn results in the divergencies in some of the physical quantities such as the scale factor, energy density and pressure profiles. As instances for these type of singularities, one may refer to Big Rip singularity [26-28], Sudden singularity [29-31] and Big Freeze singularity [32]. There are also other classifications for the possible future singularities, see for example [33-39].

The theory of conformal Weyl gravity has been proposed as an alternative theory to Einstein's GR for the high energy limit and is based on the assumption of the local conformal invariance in the geometry of spacetime $[40,41]$. There are many attempts for obtaining the Einstein's GR as the low energy limit of the conformal Weyl theory, by dynamically breaking the conformal invariance, with varying degrees of success and difficulty. In this direction, the main problem is that by breaking the conformal symmetry, the quantum fluctuations bring back the Einstein-Hilbert action with a repulsive rather than attractive gravity. However, one may refer to the recent work of Maldacena [42] where it is shown that in the four dimension, the conformal gravity with a Neumann boundary condition is classically equivalent to the Einstein gravity with a cosmological constant. It has been also suggested by Kazanas and Mannheim that the conformal Weyl gravity can be considered an independent theory on its own right instead of looking for a low energy GR limit. By this motivation, the exact vacuum solutions as well as the cosmological solutions of this theory have been found [43-46]. It is shown that in the cosmological setup, the conformal Weyl gravity can solve naturally both the cosmological constant and the flatness problems in GR . Beside these successes of the conformal Weyl gravity, it is argued that this theory fails to fulfill simultaneously the observational constraints on the present cosmological parameters and on the primordial light element abundances [47]. For the static setup of the theory, it is shown that the de Sitter space can be found as the vacuum solution of this theory and there is no need to an ad hoc introduction of the cosmological constant to the gravitational action and its corresponding field equations [43]. Moreover, in the Newtonian limit of the theory, there are also linear modification terms to the exterior Schwarzschild-de Sitter solutions that could explain the rotation curves of galaxies without any need to the mysterious dark matter $[43,48,49]$. Another argument about this theory is given in [50]. It was argued that the conformal Weyl gravity does not agree with the predictions of general relativity in the limit of weak fields and non-relativistic velocities. Nevertheless, it was counter- argued in [51], that in the presence of macroscopic long range scalar fields, the standard Schwarzschild phenomenology of GR is still recovered. In order to check the viability of the conformal Weyl gravity, the deflection of light and time delay in the exterior of a static spherically symmetric source were investigated in $[52,53]$, and it was found that the parameters of the theory fit the experimental constraints. Also, it is shown in [54] that one can find a class of wormhole geometries satisfying the energy conditions in the neighborhood of the throat of wormhole. This is in a clear contrast to the solutions in GR where exotic matter fields are needed to support a wormhole geometry.

In this work, regarding the above mentioned interesting features of the conformal Weyl gravity, we are motivated to study the issue of the past and future cosmological singularities in the context of this theory. Such a study can be also well motivated even one does not consider the Weyl gravity theory as an independent theory on its own right, but as the high energy limit alternative to GR where the being of singularities are important and inevitable issues. The organization of this paper is as follows. In Sect. 2, we review the conformal Weyl gravity theory and its cosmological field equations. In Sect. 3, we explore the existence and stability conditions for an ESU within the emergent universe scenario. In Sect. 4, we address the possible future finite-time cosmological singularities. Finally, in Sect. 5, we give our concluding remarks.

\section{Conformal Weyl gravity theory}

The action of the conformal Weyl gravity is given by

$I=-\alpha \int d^{4} x \sqrt{-g} C_{\mu \nu \rho \lambda} C^{\mu \nu \rho \lambda}+\int d^{4} x \sqrt{-g} \mathcal{L}_{M}$,

where $C_{\mu \nu \rho \lambda}$ is the Weyl tensor and $\mathcal{L}_{M}$ is the Lagrangian of the matter fields. Here, $\alpha$ is a dimensionless gravitational coupling constant. The conformal Weyl tensor and matter Lagrangian read respectively as

$$
\begin{aligned}
C_{\mu \nu \alpha \beta}= & R_{\mu \nu \alpha \beta}-g_{\mu[\alpha} R_{\beta] \mu}+\frac{1}{3} R g_{\mu[\alpha} g_{\beta] \nu}, \\
\mathcal{L}_{M}= & -\int d^{4} x \sqrt{-g}\left[\frac{1}{2} S^{\mu} S_{\mu}+\lambda S^{4}-\frac{1}{12} S^{2} R_{\mu}^{\mu}\right. \\
& \left.+i \bar{\psi} \gamma^{\mu}(x)\left(\partial_{\mu}+\Gamma_{\mu}(x)\right) \psi-h S \bar{\psi} \psi\right],
\end{aligned}
$$

where $S(x)$ is a conformal scalar field that yields symmetry breaking and makes the particles to be massive, and $\psi(x)$ is a fermion field representing the normal matter fields. Also, $\Gamma(x), R_{\mu}^{\mu}, h$ and $\lambda$ are the fermion spin connection, scalar curvature and two dimensionless coupling constants, respectively. The gravitational field equations of this theory can be 
obtained by the variation of the action (1) with respect to the metric $g_{\mu \nu}$ as [43]

$4 \alpha W_{\mu \nu}=T_{\mu \nu}$,

where $T_{\mu \nu}$ is the energy-momentum tensor corresponding to the Lagrangian of the matter fields and $W_{\mu \nu}$ is given by

$W_{\mu \nu}=W_{\mu \nu}^{(2)}-\frac{1}{3} W_{\mu \nu}^{(1)}$,

where $W_{\mu \nu}^{(1)}$ and $W_{\mu \nu}^{(2)}$ are

$$
\begin{aligned}
W_{\mu \nu}^{(1)}= & 2 g_{\mu \nu} \nabla_{\beta} \nabla^{\beta} R_{\alpha}^{\alpha}-2 \nabla_{\nu} \nabla_{\mu} R_{\alpha}^{\alpha} \\
& -2 R_{\alpha}^{\alpha} R_{\mu \nu}+\frac{1}{2} g_{\mu \nu}\left(R_{\alpha}^{\alpha}\right)^{2}, \\
W_{\mu \nu}^{(2)}= & \frac{1}{2} g_{\mu \nu} \nabla_{\beta} \nabla^{\beta} R_{\alpha}^{\alpha}+\nabla_{\beta} \nabla^{\beta} R_{\mu \nu}-\nabla_{\beta} \nabla_{\nu} R_{\mu}^{\beta} \\
& -\nabla_{\beta} \nabla_{\mu} R_{\nu}^{\beta}-2 R_{\mu \beta} R_{\nu}{ }^{\beta}+\frac{1}{2} g_{\mu \nu} R_{\alpha \beta} R^{\alpha \beta} .
\end{aligned}
$$

Due to the conformal invariance of the theory both of the $T_{\mu \nu}$ and $W_{\mu \nu}$ are kinematically traceless.

Considering the FRW metric

$d s^{2}=-d t^{2}+a^{2}(t)\left(\frac{d r^{2}}{1-k r^{2}}+r^{2} d \Omega^{2}\right)$,

which is a conformally flat metric, one finds the Friedmann equations [45]

$$
\begin{aligned}
& \rho(t)+\lambda S^{4}+\frac{S^{2}}{2}\left(\frac{\dot{a}^{2}(t)}{a^{2}(t)}+\frac{k}{a^{2}(t)}\right)=0, \\
& \rho(t)-3 p(t)+4 \lambda S^{4} \\
& +S^{2}\left(\frac{\ddot{a}(t)}{a(t)}+\frac{\dot{a}^{2}(t)}{a^{2}(t)}+\frac{k}{a^{2}(t)}\right)=0,
\end{aligned}
$$

where $\rho(t)$ and $p(t)$ are the density and pressure profiles of the perfect fluid $T_{\mu \nu}$ and $S(x)=S$ is a spacetime independent constant due to the conformal invariance of the theory. The covariant conservation of $T_{\mu \nu}$ leads to [45]

$\dot{\rho}(t)+3 H(\rho+p)=0$,

where $H=\dot{a}(t) / a(t)$ is the Hubble parameter.

In the next section, using the cosmological field Eqs. (8), (9) and (10), we study the problem of initial big bang singularity which exists in the standard model of cosmology based on Einstein's GR. We show that the conformal Weyl gravity admits the emergent universe scenario and then it can be free of the initial singularity problem.
Table 1 The existence conditions for an ESU in the context of conformal Weyl gravity

\begin{tabular}{lll}
\hline$\omega$ values & $\lambda$ values & $k$ values \\
\hline$\omega>-1 / 3$ & $\lambda>0$ & $k<0$ \\
& $\lambda<0$ & $k>0$ \\
$-1<\omega<-1 / 3$ & $\lambda>0$ & $k>0$ \\
& $\lambda<0$ & $k<0$ \\
$\omega<-1$ & $\lambda>0$ & $k<0$ \\
& $\lambda<0$ & $k>0$ \\
\hline
\end{tabular}

\section{Emergent universe paradigm: the initially non-singular state and its stability analysis}

As mentioned in the introduction, the emergent universe paradigm in GR is unstable against prevailing perturbations in very early universe and is expected to decay rapidly [9]. Therefore this scenario was developed in modified gravity theories with the hope to improve the stability at the high energy regimes. Thus, the conformal Weyl gravity as a successful theory in solving some of the fundamental problems in standard model of cosmology also deserves for studying the singularity problems in detail, which is the aim of this and the next sections.

To study the emergent universe paradigm and its stability in the context of the conformal Weyl gravity, here we consider that the perfect fluid has the barotropic equation of state $p=$ $\omega \rho$. Then, we can combine the Friedmann Eqs. (8) and (9) as the Raychadhuri equation

$\ddot{a}(t)=-\frac{1}{2}(1+3 \omega) \frac{\dot{a}^{2}(t)+k}{a(t)}-3(\omega+1) \lambda S^{2} a(t)$.

The ESU is characterized by the condition $\ddot{a}(t)=0=\dot{a}(t)$ [9]. Then, to begin with, one has to obtain the existence condition for an ESU solution in the conformal Weyl theory. The corresponding matter density and scale factor for ESU can be obtained from (8) and (11) as

$$
\begin{aligned}
& \rho_{E S}=-\lambda S^{4}-\frac{k S^{2}}{2 a_{E S}^{2}}, \\
& a_{E S}^{2}=-\frac{(1+3 \omega) k}{6(1+\omega) \lambda S^{2}} .
\end{aligned}
$$

Using (13), the existence condition for an ESU solution reduces to the reality of the corresponding scale factor $a_{E S}$. Then, one can obtain the existence conditions as they are classified in Table 1.

Then, the following points can be realized regarding (12), (13) and Table 1.

- One notes that since the scale factor of the initial ESU $a_{E S}$ has very small size, the corresponding density in (12) will 
be extremely high unless the scalar field $S$ is very small too. In turn, this represents that the matter density $\rho_{E S}$ can be arbitrary small too as the consequence of the smallness of $S$. This is one of the unique and interesting properties of the fourth order conformal Weyl gravity. The interpretation is that the Universe can born from nothing and any cosmological inhomogeneities such as those due to the large scale structures represent a perturbation around the vacuum state $T_{\mu \nu}=0$, which has mentioned also by Mannheim [45]. Indeed, this is a direct result of the vanishing rank four Weyl tensor $C_{\mu \nu \alpha \beta}$ and consequently the rank two gravitational tensor $W_{\mu \nu}$ in the field equation of conformal Weyl gravity (4) for an FRW metric. There is no such an interesting property for an FRW universe in the context of the second order Einstein's GR theory.

- In the context of Weyl gravity there exists the possibility of having ESU for the ordinary matter fields possessing the equation of state $\omega \geq 0$, and there is no need for exotic matter fields.

- The case of $\omega=-1$, representing the cosmological constant field, does not correspond to an small size initial emergent ESU for the conformal Weyl gravity theory. Indeed, as the equation of state parameter $\omega$ approaches to -1 , we have $a_{E S} \rightarrow \infty$. This fact has two meanings: (1) there is an extremely large static de Sitter limit for the theory, and (2) by approaching the equation of state parameter $\omega$ to -1 , possibly due to the perturbation in $T_{\mu \nu}$ in very early universe [45], the initial small size nonsingular Einstein static state enlarges and enters to an inflationary phase which makes hint to a natural graceful exit mechanism. At the end of this section, we will discuss this case again.

- There is no non-singular spatially flat ESU for the conformal Weyl theory, i.e., for $k=0$.

- The case of $\omega>-1 / 3, \lambda>0$ and $k<0$ has a particular importance in the sense that it can provide a solution for the flatness problem in the standard model of cosmology, see [45].

- The case $\omega=-1 / 3$ represents a singular state, i.e., $a_{E S}=0$. Then, any perturbation around $T_{\mu \nu}=0$ toward the equation of state $\omega=-1 / 3$ for matter fields yields an initially singular universe for the conformal Weyl gravity.

Now, to study the stability of ESU, we consider the Raychadhuri Eq. (11) as the following two dimensional dynamical system with the phase-space variables $x_{1}=a(t)$ and $x_{2}=\dot{a}(t)$

$$
\begin{aligned}
& \dot{x}_{1}(t)=x_{2}(t) \\
& \dot{x}_{2}(t)=-\frac{1}{2}(1+3 \omega) \frac{x_{2}^{2}(t)+k}{x_{1}(t)}-3(\omega+1) \lambda S^{2} x_{1}(t) .
\end{aligned}
$$

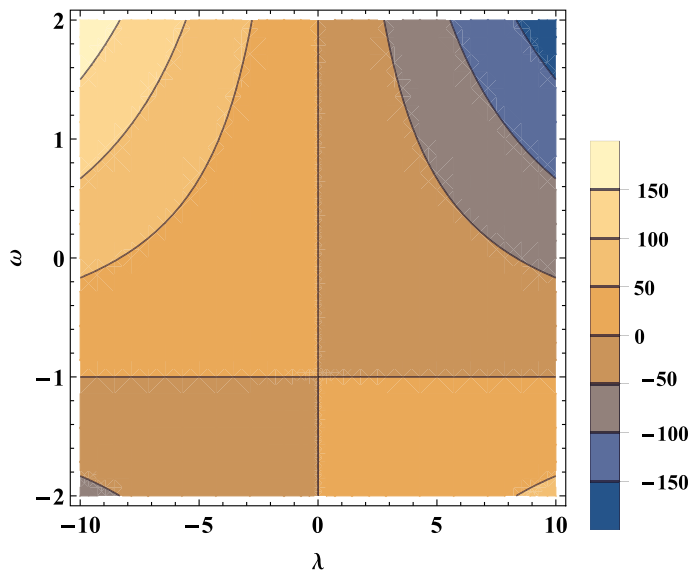

Fig. 1 The 2-dimensional contour plot for the eigenvalues $\sigma^{2}(\lambda, \omega)$ of the Jacobian matrix $J_{i j}$. The being of $\sigma^{2}<0$ regions represent the stability of the solutions

Then, for this dynamical system, ESU is corresponding to the fixed point $\left(x_{1}, x_{2}\right)=\left(a_{E S}, 0\right)$. The stability of this equilibrium point can be realized by determining eigenvalues $\sigma^{2}$ of the Jacobian matrix $J_{i j}=\left.\frac{\partial \dot{x}_{i}}{\partial x_{j}}\right|_{x_{2}=0}$. The case $\sigma^{2}>0$ represents a hyperbolic fixed point, and hence it will be unstable. For this case, the trajectories in the neighborhood of this point diverge from it. In contrast, $\sigma^{2}<0$ represents a stable center equilibrium point in which a small departure form this point results in oscillations about it. Indeed this case corresponds to the initial stable Einstein static state in which the seed universe oscillates around it indefinitely. The Jacobian matrix associated to our dynamical system (14) reads as

$J_{i j}=\left(\begin{array}{cc}0 & 1 \\ \frac{(1+3 \omega) k}{2 a_{E S}^{2}}-3(1+\omega) \lambda S^{2} & 0\end{array}\right)$,

where its eigenvalues can be obtained as

$\sigma^{2}=-6(\omega+1) \lambda S^{2}$

where we have used (13) in (16). In Fig. 1, we give the two dimensional contour plot representing $\sigma^{2}(\lambda, \omega)$. The presence of $\sigma^{2}(\lambda, \omega)<0$ regions imply the stability of the solutions.

Then, one finds that there are possible stable solutions satisfying the following stability conditions

Stability conditions: $\left\{\begin{array}{l}\omega<-1 \text { for } \lambda<0, \\ \omega>-1 \text { for } \lambda>0\end{array}\right.$

Then, regarding the conditions in Table 1 and in (17), one can conclude the required conditions guaranteing both the existence and stability of an ESU in the conformal Weyl gravity as are given in Table 2 . 
Table 2 The conditions guaranteing both of the existence and stability of an ESU in the conformal Weyl gravity theory

\begin{tabular}{lll}
\hline$\omega$ values & $\lambda$ values & $k$ values \\
\hline$\omega>-1 / 3$ & $\lambda>0$ & $k<0$ \\
$-1<\omega<-1 / 3$ & $\lambda>0$ & $k>0$ \\
$\omega<-1$ & $\lambda<0$ & $k>0$ \\
\hline
\end{tabular}

In Fig. 2, we have plotted the phase-space $(\dot{a}(t), a(t))$ diagram and the evolution of the scale factor for the corresponding closed trajectories in the phase-space. The upper plot represents some closed trajectories in the phase-space for the given typical values of the parameters satisfying the existence and stability conditions in Table 2 . In general, a closed trajectory in the phase space, as a limit cycle, represents an oscillatory system. ${ }^{1}$ The lower plot represents the evolution of the corresponding oscillatory scale factors, versus the time, to each of the closed trajectories in the phase-space. The corresponding cases are denoted by the same colors and the same set of parameters.

Until here, we have proved that the conformal Weyl gravity admits an stable ESU for the emergent universe scenario. As we addressed before, the matter density supporting this ESU can be arbitrary small, representing the possibility for being born from nothing [45]. Beside the possibility of being born from nothing for the universe, the ESU scenario in the conformal Weyl gravity has one another important theoretical advantage in comparison to the ESU in Einstein gravity as well as to some of the other modified gravity theories. This advantage is related to the fact that the de Sitter solution is a vacuum solution of the conformal Weyl theory. This vacuum solution is capable of triggering the inflation such that under the prevailing perturbations the universe can transit from the oscillations around its small size ESU to its subsequent inflationary phase and then produces whole of the cosmic history [43]. This advantage is supported by the fact that for both the FRW solution and the de Sitter solution (represented by $R_{\mu \nu}=\Lambda g_{\mu \nu}$ ), both of the Weyl tensor $C_{\mu \nu \alpha \beta}$ and the gravitational tensor $W_{\mu \nu}$ vanish. Then, as mentioned by Mannheim [45], the FRW cosmology is also the case that the energy momentum tensor is everywhere zero, and then it is another vacuum solution of the theory. Thus, any cosmological inhomogeneities such as those in the large-scale structure of the universe is a consequence of a perturbation around $T_{\mu \nu}=0$. In the presence of normal matter, such a property can be achieved nontrivially by an interplay between the various fields of the theory. In the conformal Weyl grav-

\footnotetext{
1 The differential Eq. (14) possesses the Jacobi elliptic function $x_{1}=$ $A c n(\omega t+\delta)$ as its solution which satisfies the equations $x_{1}^{\prime}=(1-$ $\left.x_{1}^{2}\right)\left(1-k_{1}^{2}+k_{1}^{2} x_{1}^{2}\right)$ and $x_{1}^{\prime \prime}=-\left(1-2 k_{1}^{2}\right) x_{1}-2 k_{1}^{2} x_{1}^{3}$ where $k_{1}$ is an arbitrary constant. In the case of $k_{1}=0$, the solution reduces to the form $x_{1}=A \cos (\omega t+\delta)$.
}
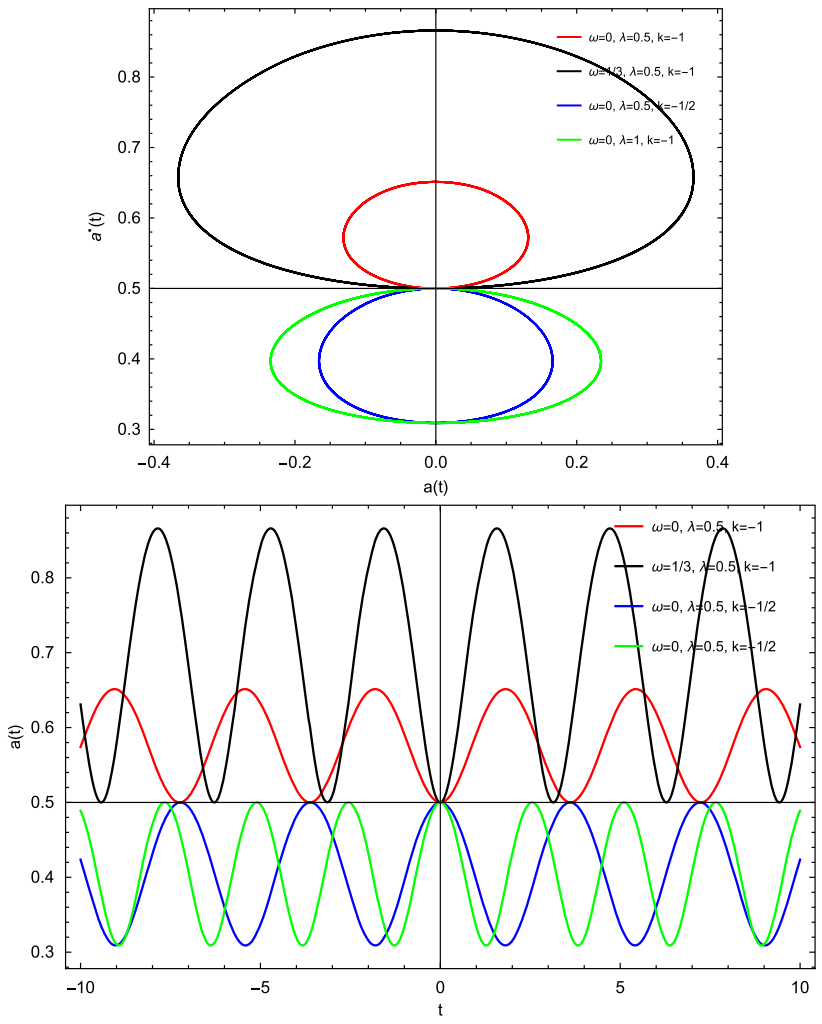

Fig. 2 The upper plot represents some closed trajectories in the phasespace $(\dot{a}(t), a(t))$ for the given typical values of the parameters satisfying the existence and stability conditions in Table 2 . The lower plot represents the evolution of the corresponding scale factors (as a function of time) to each of the closed trajectories in the phase space. The corresponding cases are in the same color

ity, the perturbations around $T_{\mu \nu}=0$ are responsible for both the generation the matter fields $(\rho(t)$ and $p(t))$ associated to the inhomogeneities in the large scale structures and the transition from one non-singular vacuum state to its subsequent another vacuum state deriving the inflation. This unique feature of having these two vacuum solutions in the conformal Weyl gravity may provides a natural mechanism for the graceful exit from the ESU to its subsequent inflationary phase and then to the radiation and matter dominated eras. We aim to study and elaborate on this important advantage of the conformal Weyl theory and report it elsewhere [55].

Before concluding this section, we consider an specific solution: a non-singular solution for $\omega=1 / 3$ and $\rho(t)=$ $\rho_{0} / a^{4}(t)$ which represents a radiation filled universe. For this case, the Friedmann Eq. (8) can be integrated which gives the following solution for the scale factor

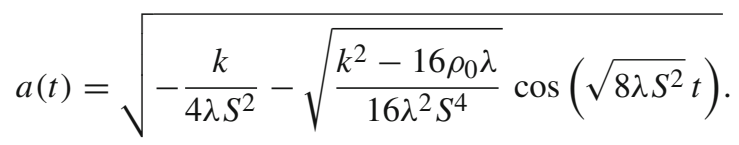


This solution represents the oscillation of the scale factor of the radiation universe between two finite minimum and maximum sizes. Then, one can interpret this solution in two different ways as

(1) An ever oscillating radiation dominated universe model with the finite minimum and maximum sizes.

(2) An eternally oscillating small size emergent universe standing as the seed of a large scale universe. In this case, the large scale universe can born and exit from this finite size state and then experience an inflationary phase by the prevailing perturbations around $T_{\mu \nu}=0$.

Believing in the history of the universe having subsequent phase transitions from the radiation and dust to the dark energy dominated phases seems to be more in the favor of the above second interpretation. This is also supported by the current observations about the accelerating expansion of the universe in the sense that the repulsive dark energy profile makes unlikely the being of a contracting phase. Here, regarding the square roots in (18), one sees that to have the oscillatory modes around the scale factor $a_{0}=\sqrt{-\frac{k}{4 \lambda S^{2}}}$, the conditions $k<0$ and $\lambda>0$ are required. These conditions are in agreement with our results for an emergent ESU given in Table 2. Also it is seen that $\rho$ should satisfy the condition $\rho_{0}<k^{2} / 16 \lambda$ which represents that the initial density of ESU state is finite for a finite and non-zero values of $k$ and $\lambda$ parameters, respectively.

\section{The possible future singularities}

In this section, following Barrow et al. [56,57], we study the possible types of finite-time singularities may happen in the future of an FRW universe in the context of the conformal Weyl gravity. To do this, based on our study in the past section for the possibility of having an initially non-singular cosmology in the context of this theory, we construct initially non-singular scale factors and then investigate the behavior of $H(t), \ddot{a}(t), \rho(t)$ and $p(t)$ versus the cosmic time.

In Table 3, we give some types of possible future singularities for an FRW universe regarding the behavior of the scale factor, matter density and pressure in a finite cosmological time [58].

Types I, II, III and IV singularities are known as "Big Rip or Cosmic Doomsday" [59,60], "Sudden" [56], "Big Freeze" [61] and "Big Brake or Big Separation" [62] singularities, respectively. In type IV singularity, although $a(t), \rho(t)$ and $p(t)$ remain finite but the higher derivatives of $H$ diverge. Following [56,57], and to keep the generality of the study in this section, we do not impose any specific equation of state on the pressure and density profiles in the field equations (8)
Table 3 The possible finite time future singularities for an FRW cosmology

\begin{tabular}{lllll}
\hline Singularity type & $t$ & $a(t)$ & $\rho(t)$ & $p(t)$ \\
\hline I & $t \rightarrow t_{s}$ & $a \rightarrow \infty$ & $\rho \rightarrow \infty$ & $|p| \rightarrow \infty$ \\
II & $t \rightarrow t_{s}$ & $a \rightarrow a_{s}$ & $\rho \rightarrow \rho_{s}$ & $|p| \rightarrow \infty$ \\
III & $t \rightarrow t_{s}$ & $a \rightarrow a_{s}$ & $\rho \rightarrow \infty$ & $|p| \rightarrow \infty$ \\
IV & $t \rightarrow t_{s}$ & $a \rightarrow a_{s}$ & $\rho \rightarrow 0$ & $|p| \rightarrow 0$ \\
\hline
\end{tabular}

and (9). We consider the solution for the scale factor $a(t)$ introduced by Barrow [56,57]

$a(t)=1+B t^{q}+C\left(t_{s}-t\right)^{n}$,

where $B, q, C$ and $n$ are positive free constants to be determined, and $t_{s}$ represents the occurring moment of the singularity. For an initially singular universe, i.e possessing the big bang singularity, one can fix the zero of time by the condition $a(0)=0$ which leads to $C=-1 / t_{s}^{n}$ and the following form for the scale factor

$a(t)=1+\left(a_{s}-1\right)\left(\frac{t}{t_{s}}\right)^{q}-\left(1-\frac{t}{t_{s}}\right)^{n}$.

where $q>0$ and $n>0$. In the present work, regarding the previous section showing the possibility of having initially non-singular universe in the context of the conformal Weyl gravity, we consider the following generalization for the above scale factor

$a(t)=1+\left(a_{s}-1\right)\left(\frac{t}{t_{s}}\right)^{q}+\left(a_{E S}-1\right)\left(1-\frac{t}{t_{S}}\right)^{n}$,

where we supposed $0<a_{E S} \ll 1<a_{S}$ and it describes a non-singular universe evolving from $a(0)=a_{E S}$, where $a_{E S}$ represents the initially non-singular Einstein static state, toward $a\left(t_{s}\right)=a_{s}$. One can simply recover the initially singular universe scenario by setting $a_{E S}=0$. Then, using (21), we obtain

$$
\begin{aligned}
\dot{a}(t)= & \frac{q\left(a_{s}-1\right)}{t_{s}}\left(\frac{t}{t_{s}}\right)^{q-1}-\frac{n\left(a_{E S}-1\right)}{t_{s}}\left(1-\frac{t}{t_{s}}\right)^{n-1} \\
\ddot{a}(t)= & \frac{q(q-1)\left(a_{s}-1\right)}{t_{s}^{2}}\left(\frac{t}{t_{s}}\right)^{q-2} \\
& +\frac{n(n-1)\left(a_{E S}-1\right)}{t_{s}^{2}}\left(1-\frac{t}{t_{s}}\right)^{n-2} .
\end{aligned}
$$

On the other hand, using the field Eqs. (8) and (9), we find the density and pressure as

$$
\begin{aligned}
& \rho(t)=-\lambda S^{4}-\frac{S^{2}}{2}\left(\frac{\dot{a}^{2}(t)}{a^{2}(t)}+\frac{k}{a^{2}(t)}\right), \\
& p(t)=\lambda S^{4}+\frac{1}{3} S^{2} \frac{\ddot{a}(t)}{a(t)}+\frac{1}{6} S^{2}\left(\frac{\dot{a}^{2}(t)}{a^{2}(t)}+\frac{k}{a^{2}(t)}\right),
\end{aligned}
$$


Here, one notes to the difference between the field equations of the fourth-order conformal Weyl gravity and the secondorder Einstein gravity. The sign differences in $\rho(t)$ and $p(t)$ is a natural result of the difference between the actions of these two theories. Using this difference in (24), it was shown by Mannheim [45] that the conformal Weyl gravity is free of the flatness problem. Another interesting feature of the conformal Weyl gravity is the coupling way of the scalar field $S$ to the dynamical quantities $a(t)$ and $\dot{a}(t)$ which can play an important role in the future singularity avoidance as we will discuss later.

Considering that the coupling $\lambda$ and the scalar field $S$ are supposed to be constant in the conformal Weyl gravity [45], we can classify the possible finite time future singularities in the context of this theory with respect to $n$ and $q$ values as in the following cases.

- The case of $t \rightarrow t_{s}$ with $0<n<1$ and $0<q \leq 1$. For this case we have

$$
\begin{aligned}
& a\left(t_{s}\right) \rightarrow a_{s}, \quad \dot{a}\left(t_{s}\right) \rightarrow+\infty, \quad H\left(t_{s}\right) \rightarrow \\
& \quad+\infty, \quad \ddot{a}\left(t_{s}\right) \rightarrow+\infty, \\
& \rho\left(t_{s}\right) \rightarrow-\infty, \quad\left|p\left(t_{s}\right)\right| \rightarrow \infty .
\end{aligned}
$$

This case represents the type III singularity as in the table (3), or Big Freeze singularity. For this case, the evolution of the scale factor $a(t)$, density $\rho(t)$ and pressure $p(t)$ are plotted in Fig. 3 for two typical values of $q$.

- The case of $t \rightarrow t_{s}$ with $n=1$ and $0<q \leq 1$.

For this case we find

$$
\begin{aligned}
& a\left(t_{s}\right) \rightarrow a_{s}, \quad \dot{a}\left(t_{s}\right) \rightarrow \dot{a}_{s}>0, \\
& \quad H\left(t_{s}\right) \rightarrow H_{s}>0, \quad \ddot{a}\left(t_{s}\right) \rightarrow \ddot{a}_{s} \leq 0 \\
& \rho\left(t_{s}\right) \rightarrow \rho_{s}<0, \quad\left|p\left(t_{s}\right)\right| \rightarrow p_{s}
\end{aligned}
$$

where all the $a_{s}, \dot{a}_{s}, H_{s}, \ddot{a}_{s}, \rho_{s}$ and $p_{s}$ are finite. Then, there is no finite-time future singularity for this case. Here, the zero acceleration case happens for $q=1$. Similarly, the evolution of the scale factor $a(t)$, density $\rho(t)$ and pressure $p(t)$ are plotted in Fig. 4 for two typical values of $q$.

- The case of $t \rightarrow t_{s}$ with $1<n<2$ and $0<q \leq 1$. For this case we have

$$
\begin{aligned}
& a\left(t_{s}\right) \rightarrow a_{s}, \quad \dot{a}\left(t_{s}\right) \rightarrow \dot{a}_{s}>0, \quad H\left(t_{s}\right) \\
& \quad \rightarrow H_{s}>0, \quad \ddot{a}\left(t_{s}\right) \rightarrow-\infty \\
& \rho\left(t_{s}\right) \rightarrow \rho_{s}<0, \quad\left|p\left(t_{s}\right)\right| \rightarrow \infty .
\end{aligned}
$$

This case represents a type II singularity, i.e. a cosmological sudden singularity. For this case, the evolution of the scale factor $a(t)$, density $\rho(t)$ and pressure $p(t)$ are plotted in Fig. 5 for typical values of $q$.

- The case of $t \rightarrow t_{s}$ with $n \geq 2$ and $0<q \leq 1$.

For this case we have

$$
\begin{aligned}
& a\left(t_{s}\right) \rightarrow a_{s}, \quad \dot{a}\left(t_{s}\right) \rightarrow \dot{a}_{s}>0, \quad H\left(t_{s}\right) \\
& \quad \rightarrow H_{s}>0, \quad \ddot{a}\left(t_{s}\right) \rightarrow \ddot{a}_{s} \leq 0 \\
& \rho\left(t_{s}\right) \rightarrow \rho_{s}<0, \quad\left|p\left(t_{s}\right)\right| \rightarrow p_{s} .
\end{aligned}
$$

where all $a_{s}, \dot{a}_{s}, H_{s}, \ddot{a}_{s}, \rho_{s}$ and $p_{s}$ are finite. Then, for this case all the physical quantities remain finite. The evolution of the scale factor $a(t)$, density $\rho(t)$ and pressure $p(t)$ are plotted in Fig. 6 for two typical values of $q$. Then, we see that for an initially non-singular universe which evolves according to (21), in general there are type II and III cosmological singularities in the context of conformal Weyl gravity. The type IV singularity, i.e $t \rightarrow t_{s}, a \rightarrow a_{s}, \rho \rightarrow 0$ and $|p| \rightarrow 0$, in the context of this theory with the scale factor (21) can be achieved for the very small values of the scalar field $S$ values $(S \rightarrow 0)$ such that $S \dot{a}_{s} \rightarrow 0$ and $S^{2} \ddot{a}_{S} \rightarrow 0$. Even in the cases of divergent $\dot{a}(t)$ and $\ddot{a}(t)$, the limiting values of $S \dot{a}_{S} \rightarrow 0$ and $S^{2} \ddot{a}_{S} \rightarrow 0$ can be obtained as the result of $0 \times \infty$ type limits which require $\frac{\ddot{a}(t)}{\dot{a}^{2}(t)}$ and $\frac{\dddot{a}(t)}{\ddot{a}^{2}(t)}$ to be finite, respectively. Finally, for the scale factor (21), for $0 \leq t \leq t_{s}$ we have $a_{E S} \leq a(t) \leq a_{S}$, then the big rip singularity defined as $t \rightarrow t_{s}, a \rightarrow \infty, \rho \rightarrow \infty$ and $|p| \rightarrow \infty$ can not be achieved for a finite time. However, one may consider the scale factor

$a(t)=a_{E S}\left(1+\frac{t}{t_{S}-t}\right)^{n}$,

for a universe evolving from an initial non-singular state $a_{E S}$ where $0 \leq t \leq t_{s}$ and $n \geq 1$. Then, through the field Eqs. (24) and (25), for $t \rightarrow t_{s}$, we find $a \rightarrow \infty, \rho \rightarrow$ $-\infty$ and $|p| \rightarrow \infty$ representing a Big Rip type singularity.

We conclude our analysis for the possible future cosmological singularities in the context of the conformal Weyl gravity in the following two points.

(1) In general, there are possibilities for having the types II, III and I cosmological singularities for an initially non-singular universe evolving according to the scale factors (21) and (34), respectively. The type IV singularity requires that the scalar field $S$ takes very small values and $\frac{\ddot{a}(t)}{\dot{a}^{2}(t)}$ and $\frac{\dddot{a}(t)}{\ddot{a}^{2}(t)}$ to be finite. Then, the final fate of the cosmos in the conformal Weyl gravity can be a sudden, big freeze, big rip or big brake singularity depending on the evolution of the scale factor $a(t)$ and scalar field $S$ value. 

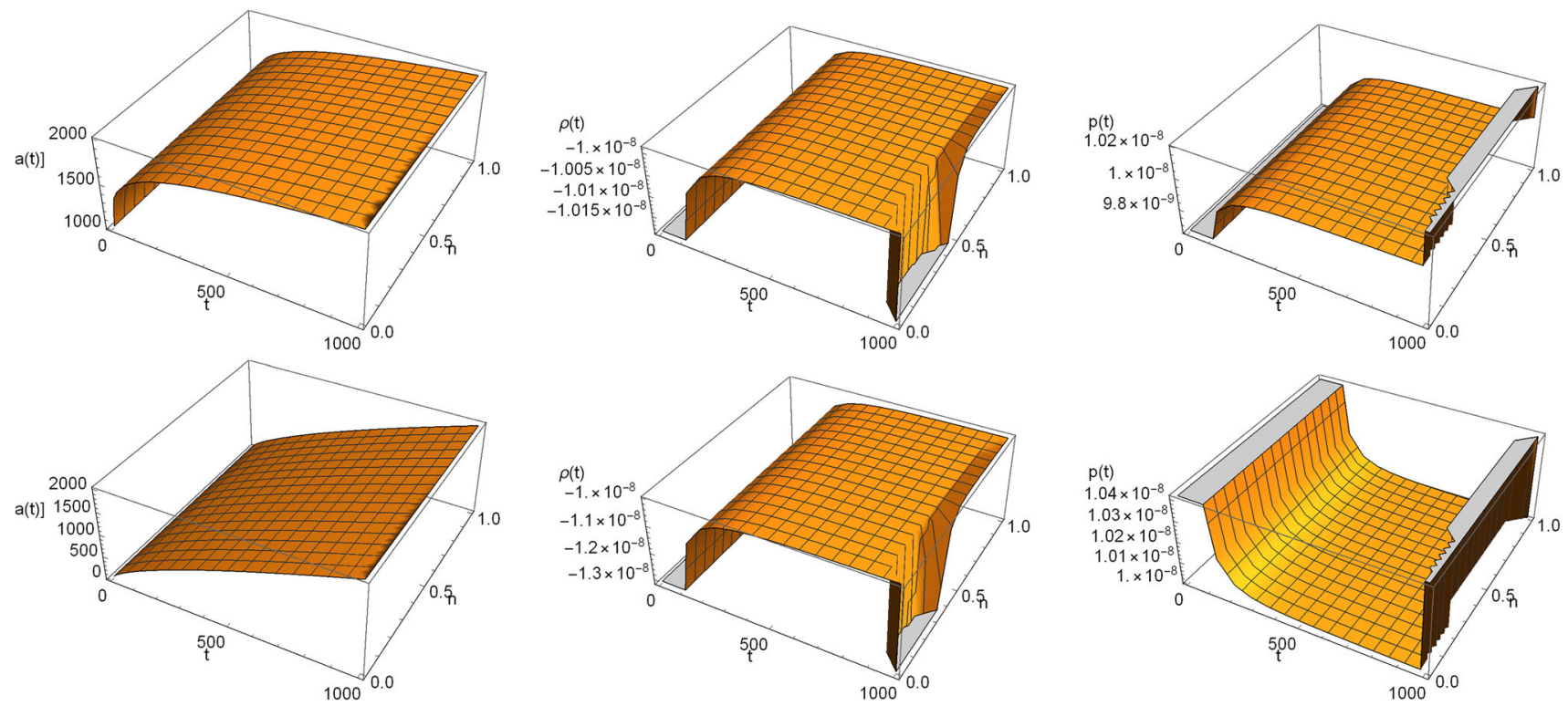

Fig. 3 The evolution of the scale factor $a(t)$, density $\rho(t)$ and pressure $p(t)$ for typical values of $q=0.2$ in the upper plot and $q=0.8$ in the lower plot. We have set $a_{s}=2000, a_{E S}=0.01, t_{s}=1000, \lambda=$ $1, S=0.01$ and $k=-1$. We see that by increasing $q$ value, the slopes

of the $\rho$ and $p$ plots for $t \rightarrow t_{s}$ is increasing which means $\rho$ and $p$ blowing up rapidly. The initial apparent over-density and pressure in the lower plots are due to the smallness of $a_{E S}$
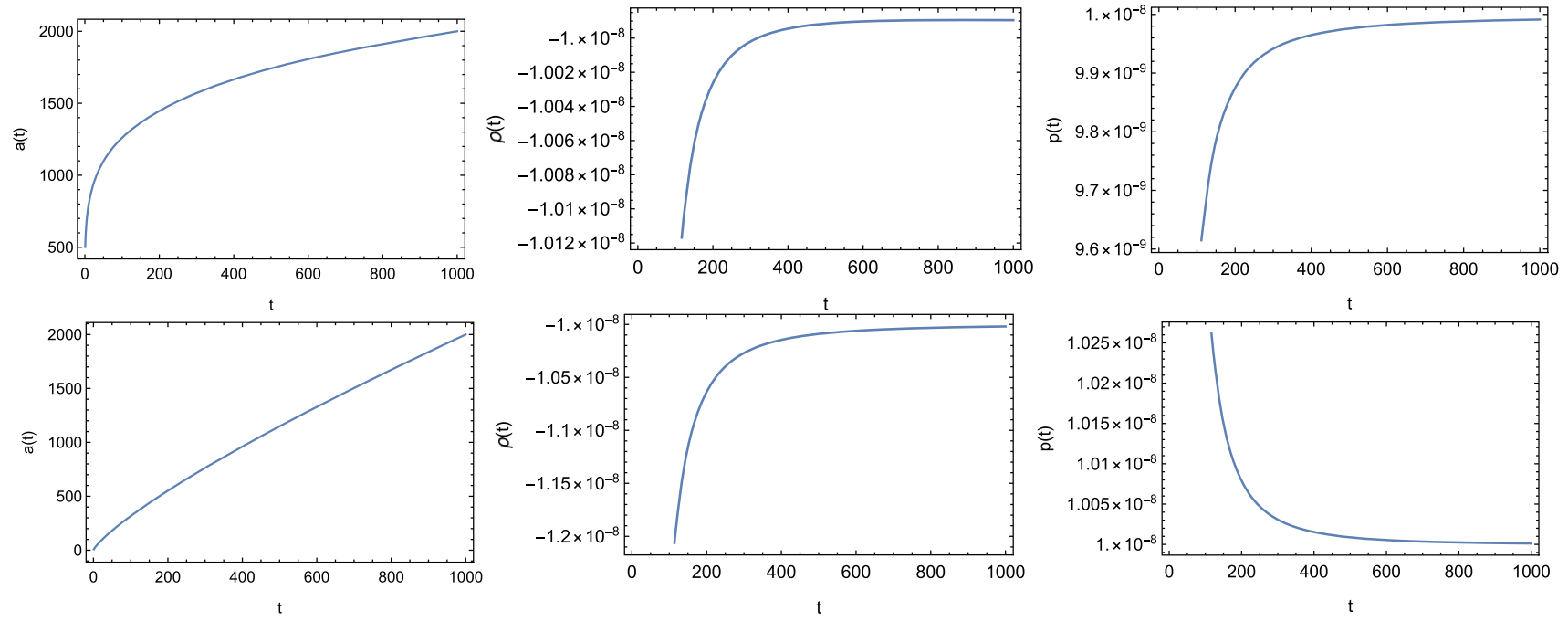

Fig. 4 The evolution of the scale factor $a(t)$, density $\rho(t)$ and pressure $p(t)$ for typical values of $q=0.1$ in the upper plot and $q=0.8$ in the lower plot. We have set $a_{s}=2000, a_{E S}=0.01, t_{s}=1000, \lambda=$
$1, S=0.01$ and $k=-1$. It is seen that by increasing $q$ value, the slopes of the $\rho$ and $p$ plots for $t \rightarrow t_{S}$ is increasing. The initial over-density and pressure are due to the smallness of $a_{E S}$
(2) The conformal Weyl gravity possesses one interesting property: the unique type of coupling the scalar field $S$ to the dynamical quantities $a(t), H(t)$, and $\ddot{a}(t)$. Indeed, these dynamical quantities are the origins of various type of future cosmological singularities. For the singularities that the divergencies in $\rho(t)$ and $p(t)$ are due to the divergencies in $H$ and $\ddot{a}(t)$, one may consider the following finite-time fine-tunings: $S H\left(t_{s}\right) \rightarrow$ finite and
$S \ddot{a}\left(t_{s}\right) \rightarrow$ finite. Then, by these fine-tunings, the scalar field $S$ can control and regularize the corresponding singularities and makes free the conformal theory from any singularity problem. The smallness of $S$ as a result of being the FRW solution as the vacuum solution for the conformal Weyl theory guarantees the avoidance of finite time future singularities. There is no such a possibility in Einstein's GR for a scalar field as the matter source of 

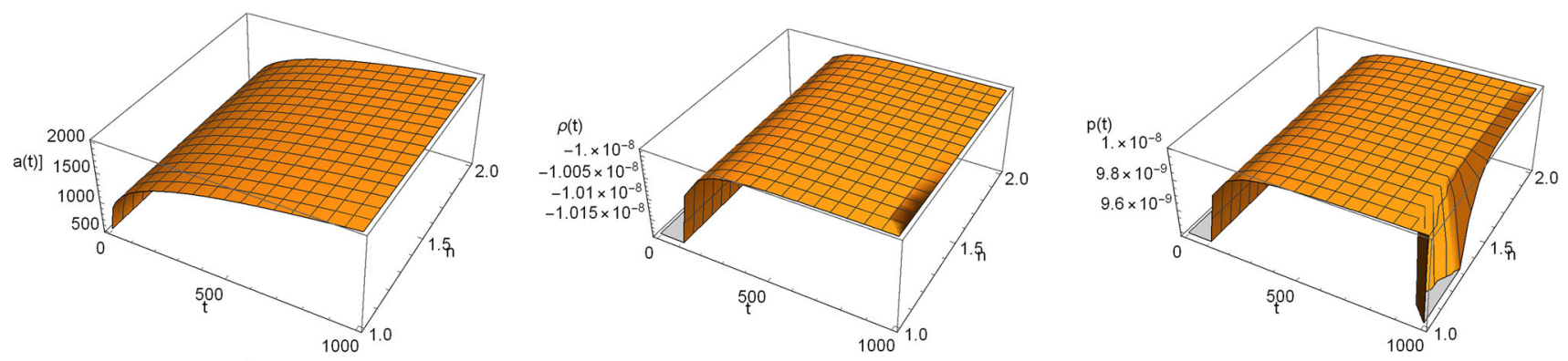
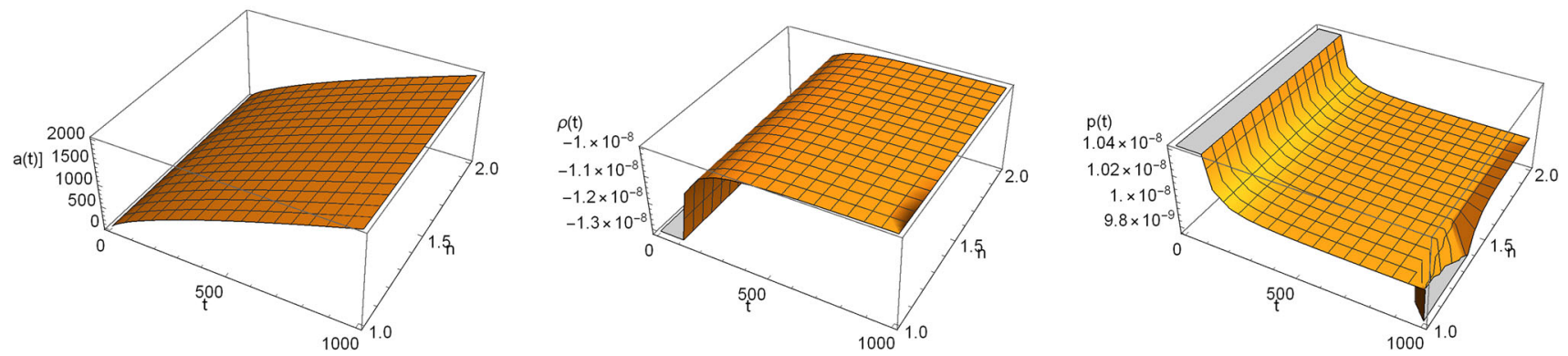

Fig. 5 The evolution of the scale factor $a(t)$, density $\rho(t)$ and pressure $p(t)$ for typical values of $q=0.2$ in the upper plot and $q=0.8$ in the lower plot. We have set $a_{s}=2000, a_{E S}=0.01, \quad t_{s}=1000, \lambda=$
$1, \quad S=0.01$ and $k=-1$. By increasing $q$ value, $\rho$ and $p$ increase more rapidly for $t \rightarrow t_{s}$. The initial over-density and pressure in the lower plots are due to the smallness of $a_{E S}$ the field equations. Then, the conformal Weyl gravity can provide a cosmological model which is free of both the past and future singularities.

\section{Conclusion}

In the present work, we studied the issue of the past and future cosmological singularities in the context of the fourth-order conformal Weyl gravity. For the past singularity problem, we investigated the emergent universe scenario proposed by Ellis et al. We obtained the stability conditions for the corresponding ESU using the fixed point approach. We showed that depending on the values of the parameters of the conformal Weyl gravity theory, there are possibilities for having initially stable ESUs for an FRW universe with both the positive and negative spatial curvatures. In particular, it is found that there exists the possibility of having ESU for the ordinary matter fields possessing the equation of state $\omega \geq 0$, but the conformal Weyl theory is not capable to have a non-singular spatially flat ESU. In contrast to GR, here we found that the matter density of the initial ESU, i.e $\rho_{E S}$, can be arbitrary small which represents the possibility of being born form nothing. Moreover, the fact that both the FRW solution and de Sitter solution are vacuum solutions of the conformal Weyl theory provides a unique feature for this theory in the sense that the initially non-singular emergent universe can gracefully exit from one vacuum to the other vacuum solution. We discussed that by approaching the equation of state parameter $\omega$ to -1 , possibly due to the perturbation in $T_{\mu \nu}=0$ in the early universe [45], the initial small size non-singular ESU enlarges and enters to an inflationary phase. In the conformal Weyl theory, the perturbations around $T_{\mu \nu}=0$ are responsible for both the generation of the matter fields $(\rho(t)$ and $p(t))$ associated to the inhomogeneities in the large scale structures and for the transition from one non-singular vacuum state to its subsequent another vacuum state deriving the inflation. This unique feature of having these two vacuum solutions in the conformal Weyl gravity may provides a natural mechanism for a graceful exit from the ESU to inflationary phase and then to its subsequent radiation and matter dominated eras. We aim to study and elaborate on this point and report such a possibility elsewhere [55].

Then, following Barrow et al., we addressed the possible types of the finite-time future cosmological singularities such as Big Rip, Sudden, Big Freeze and Big Brake singularities. We discussed that these future cosmological singularities can be the fate of the universe in the context of this theory in a general setup. However, the conformal Weyl gravity possesses one interesting property: the unique type of coupling of the scalar field $S$ to the dynamical quantities $a(t), H(t)$, and $\ddot{a}(t)$. Indeed, these dynamical quantities are the origins of various type of future cosmological singularities. For the singularities that the divergencies in $\rho(t)$ and $p(t)$ are due to the divergencies in $H(t)$ and $\ddot{a}(t)$, by considering the finitetime fine-tunings $S H\left(t_{s}\right) \rightarrow$ finite and $S \ddot{a}\left(t_{s}\right) \rightarrow$ finite, the scalar field $S$ can control and regularize the corresponding singularities and make free the conformal Weyl theory from 

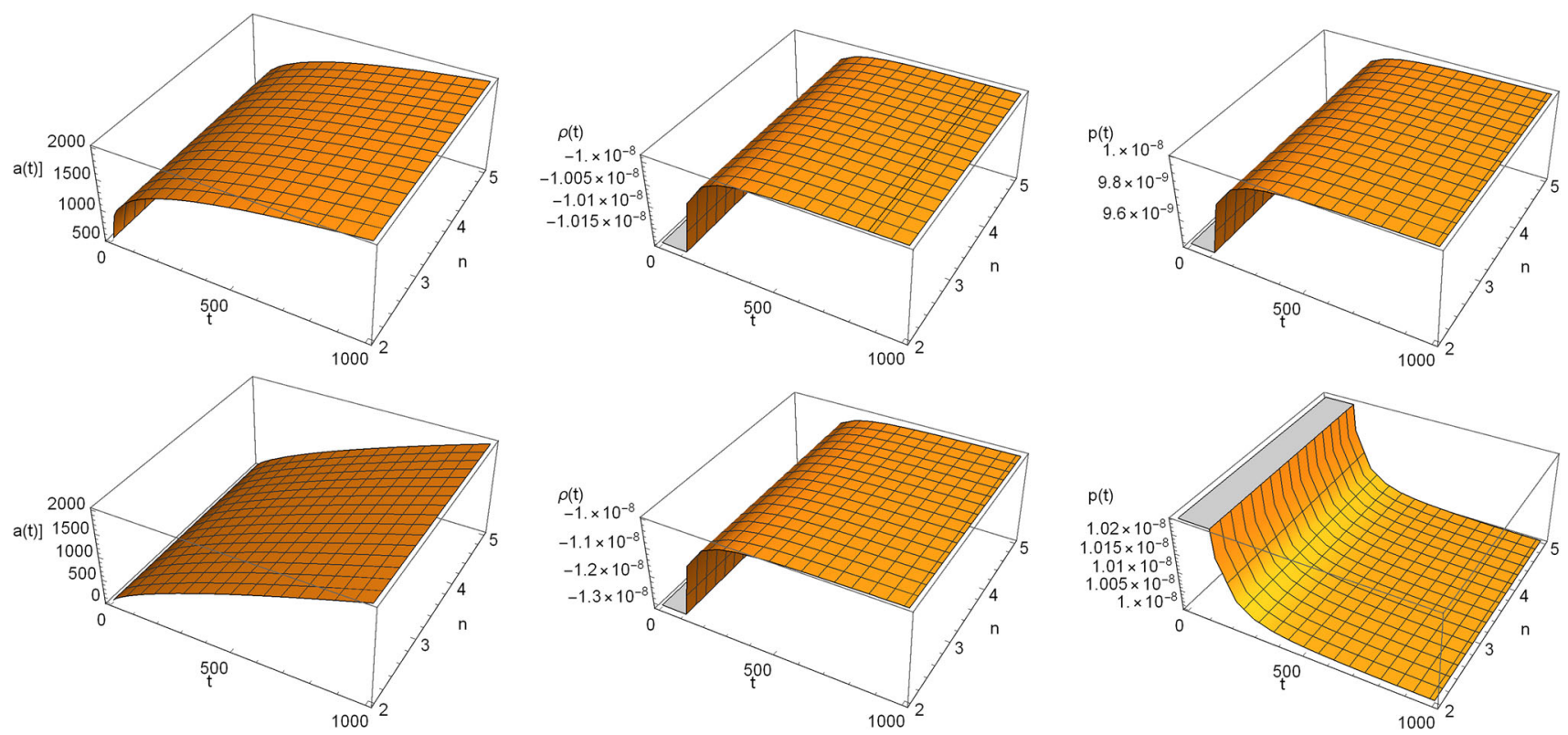

Fig. 6 The evolution of the scale factor $a(t)$, density $\rho(t)$ and pressure $p(t)$ for typical values of $q=0.2$ in the upper plot and $q=0.8$ in the lower plot. We have set $a_{s}=2000, a_{E S}=0.01, \quad t_{s}=1000, \lambda=$

$1, \quad S=0.01$ and $k=-1$. By increasing $q$ value, $\rho$ and $p$ increase rapidly for $t \rightarrow t_{s}$. The initial over-density and pressure in the lower plots are due to the smallness of $a_{E S}$

any singularity problem. The smallness of $S$ as a result of being the FRW solution as the vacuum solution for the conformal Weyl theory guarantees the avoidance of finite time future singularities. There is no such a possibility in Einstein's GR for a scalar field as the matter source of the field equations. Then, the conformal Weyl gravity can provide a cosmological model which is free of both the past and future singularities.

Acknowledgements The author deeply thanks Prof. Metin Gürses for useful comments.

Data Availability Statement This manuscript has no associated data or the data will not be deposited. [Authors' comment: Concerning the initial and final state cosmological singularities, there is no available data for this theoretical work.]

Open Access This article is distributed under the terms of the Creative Commons Attribution 4.0 International License (http://creativecomm ons.org/licenses/by/4.0/), which permits unrestricted use, distribution, and reproduction in any medium, provided you give appropriate credit to the original author(s) and the source, provide a link to the Creative Commons license, and indicate if changes were made. Funded by SCOAP ${ }^{3}$.

\section{References}

1. S.W. Hawking, R. Penrose, The Singularities of gravitational collapse and cosmology. Proc. Roy. Soc. Lond. A 314, 529 (1970)

2. A. Borde, A. Vilenkin, Eternal inflation and the initial singularity. Phys. Rev. Lett. 72, 3305 (1994)
3. M. Novello, S.E.P. Bergliaffa, Bouncing cosmologies. Phys. Rep. 463, 127 (2008)

4. Y.F. Cai, T. Qiu, X. Zhang, Y.S. Piao, and M. Li, Bouncing universe with Quintom matter, JHEP10, 071 (2007)

5. C. Lin, R.H. Brandenberger, L. Perreault Levasseur, A matter bounce by means of ghost condensation JCAP04, 019 (2011)

6. G.F.R. Ellis, R. Maartens, The emergent universe: inflationary cosmology with no singularity. Class. Quantum Grav. 21, 223 (2004)

7. G.F.R. Ellis, J. Murugan, C.G. Tsagas, The emergent universe: an explicit construction. Class. Quantum Grav. 21, 233 (2004)

8. A.S. Eddington, On the instability of Einstein's spherical world. Mon. Not. R. Astron. Soc. 90, 668 (1930)

9. J.D. Barrow, G.F.R. Ellis, R. Maartens, C.G. Tsagas, On the stability of the Einstein static universe. Class. Quant. Grav. 20, L155 (2003)

10. D.J. Mulryne, R. Tavakol, J.E. Lidsey, G.F.R. Ellis, An emergent universe from a loop. Phys. Rev. D 71, 123512 (2005)

11. L. Parisi, M. Bruni, R. Maartens, K. Vandersloot, The Einstein static universe in loop quantum cosmology. Class. Quantum Grav. 24, 6243 (2007)

12. C.G. Bohmer, L. Hollenstein, F.S.N. Lobo, Stability of the Einstein static universe in $f(R)$ gravity. Phys. Rev. D 76, 084005 (2007)

13. N. Goheer, R. Goswami, P.K.S. Dunsby, Dynamics of $f(R)$ cosmologies containing Einstein static models Class. Quant. Grav. 26, 105003 (2009)

14. M. Khodadi, Y. Heydarzade, F. Darabi, E.N. Saridakis, Emergent universe in Horava-Lifshitz-like F(R) gravity. Phys. Rev. D 93, 124019 (2016)

15. P. Wu, H. Yu, The stability of the Einstein static state in $f(T)$ gravity. Phys. Lett. B 703, 223 (2011)

16. J.T. Li, C.C. Lee, C.Q. Geng, Einstein static universe in exponential $f(T)$ gravity. Eur. Phys. J. C 73, 2315 (2013)

17. H. Shabani, A.H. Ziaie, Stability of the Einstein static Universe in $f(R, T)$ gravity. Eur. Phys. J. C 77, 31 (2017) 
18. C.G. Bohmer, The Einstein static universe with torsion and the sign problem of the cosmological constant. Class. Quant. Grav. 21, 1119 (2004)

19. K. Atazadeh, Stability of the Einstein static universe in EinsteinCartan theory. JCAP 06, 020 (2014)

20. J.E. Lidsey, D.J. Mulryne, Graceful entrance to braneworld inflation. Phys. Rev. D 73, 083508 (2006)

21. K. Atazadeh, Y. Heydarzade, F. Darabi, Einstein static universe in Braneworld scenario. Phys. Lett. B 732, 223 (2014)

22. Y. Heydarzade, F. Darabi, Induced matter brane Gravity and Einstein static universe. JCAP 1504(04), 028 (2015)

23. L. Parisi, N. Radicella, G. Vilasi, Stability of the Einstein static universe in massive gravity. Phys. Rev. D 86, 024035 (2012)

24. M. Mousavi, F. Darabi, On the stability of Einstein static universe at background level in massive bigravity. Nucl. Phys. B 919, 523 (2017)

25. S. Carneiro, R. Tavakol, Stability of the Einstein static universe in presence of vacuum energy. Phys. Rev. D 80, 043528 (2009)

26. R.R. Caldwell, A phantom menace? Cosmological consequences of a dark energy component with super-negative equation of state. Phys. Lett. B 545, 23 (2002)

27. R.R. Caldwell, M. Kamionkowski, N.N. Weinberg, Phantom energy: dark energy with $\omega<-1$ causes a cosmic doomsday. Phys. Rev. Lett. 91, 071301 (2003)

28. S. Nojiri, S.D. Odintsov, S. Tsujikawa, Properties of singularities in the (phantom) dark energy universe. Phys. Rev. D 71, 063004 (2005)

29. J.D. Barrow, Sudden future singularities. Class. Quantum Grav. 21, L79 (2004)

30. J.D. Barrow, Ch. Tsagas, New isotropic and anisotropic sudden singularities. Class. Quantum Grav. 22, 1563 (2005)

31. k. Lake, Sudden future singularities in FLRW cosmologies, Class. Quantum Grav. 21, L129 (2004)

32. M. Bouhmadi-Lopez, P.F. Gonzalez-Diaz, P. Martin-Moruno, Worse than a big rip? Phys. Lett. B 659, 1 (2008)

33. M.P. Dabrowski, T. Denkiewicz, Exotic-singularity-driven dark energy. AIP Conf. Proc. 1241, 561 (2010)

34. K. Bamba, S. Nojiri, S.D. Odintsov, The universe future in modified gravity theories: approaching the finite-time future singularity. J. Cosmol. Astropart. Phys. 45, (2008). arXiv:0807.2575 [hep-th]

35. K. Bamba, S.D. Odintsov, L. Sebastiani, S. Zerbini, Finite-time future singularities in modified Gauss-Bonnet and $\mathcal{F}(\mathrm{R}, \mathrm{G})$ gravity and singularity avoidance. Eur. Phys. J. C 67, 295-310 (2010)

36. S. Nojiri, S.D. Odintsov, Future evolution and finite-time singularities in $\mathrm{F}(\mathrm{R})$ gravity unifying inflation and cosmic acceleration. Phys. Rev. D 78, 046006 (2008)

37. F. Briscese, E. Elizalde, S. Nojiri, S.D. Odintsov, Phantom scalar dark energy as modified gravity: understanding the origin of the Big Rip singularity. Phys. Lett. B 646, 105-111 (2007)

38. S. Capozziello, M. De Laurentis, S. Nojiri, S.D. Odintsov, Classifying and avoiding singularities in the alternative gravity dark energy models. Phys. Rev. D 79, 124007 (2009)

39. S. Nojiri, S.D. Odintsov, Quantum escape of sudden future singularity. Phys. Lett. B 595, 1-8 (2004)

40. S.L. Adler, Einstein gravity as a symmetry-breaking effect in quantum field theory Rev. Mod. Phys. 54, 729 (1982)

41. A. Zee, Einstein gravity emerging from quantum Weyl gravity. Ann. Phys. 151, 431 (1983)

42. J. Maldacena, Einstein gravity from conformal gravity, arXiv: 1105.5632
43. P.D. Mannheim, D. Kazanas, Exact vacuum solution to conformal Weyl gravity and galactic rotation curves. Astrophys. J. 342, 635 (1989)

44. D. Kazanas, P.D. Mannheim, General structure of the gravitational equations of motion in conformal weyl gravity. Astrophys. J. Suppl. 76, 431 (1991)

45. P.D. Mannheim, Conformal gravity and the flatness problem. Astrophys. J. 391, 429 (1992)

46. P.D. Mannheim, Conformal cosmology with no cosmological constant. Gen. Rel. Grav. 22, 289 (1990)

47. D. Elizondo, G. Yepes, Can conformal Weyl gravity be considered a viable cosmological theory? Astrophys. J. 428, 17 (1994)

48. P.D. Mannheim, Attractive and Repulsive Gravity. Found. Phys. 30, 709 (2000)

49. A. Edery, A.A. Methot, M.B. Paranjape, Gauge choice and geodetic deflection in conformal gravity. Gen. Rel. Grav. 33, 2075 (2001)

50. E.E. Flanagan, Fourth order Weyl gravity. Phys. Rev. D 74, 023002 (2006)

51. P.D. Mannheim, Schwarzschild limit of conformal gravity in the presence of macroscopic scalar fields. Phys. Rev. D 75, 124006 (2007)

52. A. Edery, M.B. Paranjape, Classical tests for Weyl gravity: deflection of light and radar echo delay. Phys. Rev. D 58, 024011 (1998)

53. S. Pireaux, Light deflection in Weyl gravity: critical distances for photon paths. Class. Quant. Grav. 21, 1897 (2004)

54. F.S.N. Lobo, General class of wormhole geometries in conformal Weyl gravity. Class. Quant. Grav. 25, 175006 (2008)

55. Y. Heydarzade, Graceful exit in conformal Weyl theory (in preparation)

56. J.D. Barrow, Sudden future singularities. Class. Quantum Grav 21, L79 (2004)

57. J.D. Barrow, More general sudden singularities. Class. Quantum Grav 21, 5619 (2004)

58. S. Nojiri, S.D. Odinstov, S. Tsujikawa, Properties of singularities in the (phantom) dark energy universe. Phys. Rev. D 71, 063004 (2005)

59. R.R. Caldwell, A phantom menace? Cosmological consequences of a dark energy component with super-negative equation of state. Phys. Lett. B. 545, 23 (2002)

60. R.R. Caldwell, M. Kamionkowski, N.N. Weinberg, Phantom Energy: Dark Energy with $\omega<-1$ Causes a Cosmic Doomsday. Phys. Rev. Lett 91(7), 071301 (2003)

61. S. Nojiri, S.D. Odintsov, Final state and thermodynamics of a dark energy universe. Phys. Rev. D 70, 103522 (2004)

62. V. Gorini, AYu. Kamenshchik, U. Moschella, V. Pasquier, Tachyons, scalar fields, and cosmology. Phys. Rev. D 69, 123512 (2004) 